\title{
Water Effect Trial
}

National Cancer Institute

\section{Source}

National Cancer Institute. Water Effect Trial. NCI Thesaurus. Code C161480.

A type of trial designed to evaluate the effects of water on investigational product safety and/or efficacy. 